\title{
ON THE COMPLEXITY OF RATIONALIZING BEHAVIOR
}

\author{
JOSE APESTEGUIA ${ }^{\dagger}$ AND MIGUEL A. BALLESTER ${ }^{\ddagger}$
}

\begin{abstract}
We study the complexity of rationalizing choice behavior. We do so by analyzing two polar cases, and a number of intermediate ones. In our most structured case, that is where choice behavior is defined in universal choice domains and satisfies the "weak axiom of revealed preference," finding the complete preorder rationalizing choice behavior is a simple matter. In the polar case, where no restriction whatsoever is imposed, either on choice behavior or on choice domain, finding the complete preorders that rationalize behavior turns out to be intractable. We show that the task of finding the rationalizing complete preorders is equivalent to a graph problem. This allows the search for existing algorithms in the graph theory literature, for the rationalization of choice.
\end{abstract}

Keywords: Rationalization, Computational complexity, NP-complete, Arbitrary choice domains.

JEL classification numbers: D00.

\section{InTRODUCTION}

The theory of individual decision making is typically formulated on the basis of two different approaches. A revealed preference approach that directly studies individual choice behavior through choice correspondences. Or a preference relation approach, where tastes are summarized through binary relations. Clearly, the relation between these two distinct formal approaches to individual behavior is a fundamental question in economics. It is said that a collection of preference relations rationalizes choice behavior, whenever both approaches identify the same alternatives for every possible choice problem

Date: August, 2007.

* This paper supercedes a previous paper with the title "Minimal Books of Rationales." We thank Jon Benito, Ricard Gavalda, Ignacio Palacios-Huerta, Xavier Vila, an anonymous referee, and especially Coralio Ballester for his key contribution to the proof of Theorem 3.2. Financial support from Ministerio de Ciencia y Tecnología through grants SEJ2006-11510, SEJ2005-01481/ECON and FEDER, Generalitat de Catalunya through grant 2005SGR00454 and Barcelona Economics-CREA is gratefully acknowledged.

${ }^{\dagger}$ Universitat Pompeu Fabra. E-mail: jose.apesteguia@upf .edu.

†Universitat Autònoma de Barcelona. E-mail: mballester@pareto.uab.es. 
that may arise. ${ }^{1}$ This problem has attracted a great deal of attention in the literature.

Drawing on the tools of theoretical computer science, we study the question of how complex it is to find the preference relations that rationalize choice behavior. That is, given any possible choice rule, how difficult it is to construct the collection of preference relations that summarizes choice behavior. In spite of the fact that the question of rationalization is central to economics, to the best of our knowledge this paper is the first one to study the practical difficulty of rationalization.

We analyze two polar cases and some intermediate ones. In the first place we study what we call the rational procedure. This is the case where the choice correspondence satisfies the well-known consistency property known as the "weak axiom of revealed preference" (WARP). The classic result in this case is that a choice correspondence defined on the universal choice domain (i.e., choice is defined on all possible choice problems) satisfying WARP is rationalized by a unique complete preorder. We show that finding the complete preorder in such a case is a simple matter, as there are algorithms that can easily construct it.

We then turn the analysis to the polar case where no restriction whatsoever is imposed, neither on choice behavior nor on the choice domain. In other terms, we do not impose any consistency property on choice behavior; neither WARP, nor any other property. With regard to the choice domain, we consider arbitrary collections of choice problems. Then, drawing from a seminal paper by Kalai, Rubinstein, and Spiegler (2002; hereafter KRS), in order to rationalize behavior we use a collection (a book) of complete preorder relations (rationales), such that for every choice problem $A$ in the domain of feasible choice problems, the choice $c(A)$ is maximal in $A$ for some complete preorder. Clearly, there are multiple books that rationalize a given choice rule. KRS naturally propose to focus on those books that use the minimal number of preference relations. In this case, we show that, contrary to the previous one, finding a minimal book is a difficult computational problem (see Theorem 3.2). That is, there is little hope for the existence of an algorithm that for every possible choice rule finds a minimal book in a reasonable time frame. Hence, in this extremely unstructured case, the task of finding the collection of preferences that rationalize behavior is in general intractable.

Now, the question arises whether it is the conjunction of (i) unstructured choice behavior and (ii) unrestricted choice domain that leads to the computational hardeness of the problem of rationalization. It could be the case

\footnotetext{
${ }^{1}$ In the next section we will make precise the terminology we use in this introduction.
} 
that the difficulty in finding the preference relations rationalizing choice comes from the unstructured nature of behavior. It turns out that the answer to this question seems to depend on the single or multi-valued nature of the choice function.

Theorem 3.4 suggests that in the case of single-valued choice functions, the essence of the intractability of rationalization is triggered by the interplay of both unstructured behavior and unrestricted domain. Theorem 3.4 shows that under the universal choice domain, the problem of finding a minimal book is quasi-polynomially bounded. However, we argue that in the case of choice correspondences the practical difficulty of rationalizing behavior may be due to the nature of choice behavior per se.

The challenge is then to understand the driving force of the complexity of rationalization. If we are able to understand the roots of the complexity in rationalizing choice behavior, we may use this to search for specific algorithms that behave well under certain circumstances. We start this challenge by defining two binary relations on the space of choice problems, that capture two fundamental properties on the structural relation of choice problems. By doing so we will be able to draw a connection with a graph theory problem (see Theorem 4.3). This is especially useful since there is a wealth of algorithms for graph problems that may be used to solve the problem of rationalization of certain choice structures.

We then end the analysis by exploring a specific case, inspired by the recent work of Manzini and Mariotti (2007; hereafter MM). MM study the nature of choice functions that can be rationalized by sequentially applying a fixed set of asymmetric binary relations. Among other results, they provide a full characterization for the case when a choice function is sequentially rationalizable by two rationales. Such a choice function is called a Rational Shortlist Methods (RSMs). Using the tools derived for establishing the connection with graph theory, we show that the rationalization of RSMs through multiple rationales turns out to be a computationally tractable problem.

Apart from those papers already mentioned, Salant (2003), Ok (2005), and $\mathrm{Xu}$ and Zhou (2007) constitute recent related works. Salant (2003) studies two computational aspects of choice when the "independence of irrelevant alternatives" (IIA) axiom does not necessarily hold: the amount of memory choice behavior requires, and the computational power needed for the computation of choice. He shows that the rational procedure is favored by these considerations. Ok (2005) provides an axiomatic characterization of choice correspondences that satisfy the IIA axiom. Finally, Xu and Zhou (2007) propose the rationalization through extensive games with perfect information, 
and provide a full characterization of those choice functions that can be so rationalized.

The rest of the paper is organized as follows. Section 2 gives the definitions on choice behavior and binary relations, and makes precise the notion of rationalization we will use throughout the paper. Furthermore, it contains a brief introduction to the theory of NP-completeness. Section 3 contains the complexity results. In section 4 we draw on the connection between the problem of rationalization and the literature on graph theory. Finally, section 5 concludes and relates our work to the economics literature on language and to the bounded rationality literature.

\section{Preliminaries}

2.1. Choice behavior and preference relations. Let $X$ be a finite set of $n$ objects. We denote by $\mathcal{U}$ the set of all non-empty subsets of $X$. We also consider the general case of arbitrary domains $\mathcal{D} \subseteq \mathcal{U}$, with $\mathcal{D} \neq \emptyset$. A choice correspondence $c$ on $\mathcal{D}$ assigns to every $A \in \mathcal{D}$ a non-empty set of elements $c(A) \subseteq A$. A single-valued choice function simply assigns to every $A \in \mathcal{D}$, a unique element $c(A) \in A .^{2}$

The weak axiom of revealed preference is the classic consistency property:

Weak Axiom of Revealed Preference (WARP): Let $A, B \in \mathcal{D}$ and assume $x, y \in A \cap B$; if $x \in c(A)$ and $y \in c(B)$, then we must also have $x \in c(B)$.

WARP imposes a great deal of structure on choice. This can be best appreciated through its implications on the connection with preference relations. In order to elaborate on the connection between choice behavior and preference relations, which is central to this paper, we first introduce some notation. Denote by $\succeq$ a binary relation on $X, \succeq \subseteq X \times X$. Binary relations $\succ$ and $\sim$ are the asymmetric and symmetric parts of $\succeq$, respectively. Hence, $x \succ y$ if and only if $x \succeq y$ and $\neg(y \succeq x)$, and $x \sim y$ if and only if $x \succeq y$ and $y \succeq x$. We will say that a binary relation is a preorder if it is reflexive and transitive. We will often refer to complete preorders by rationales. We will say that a binary relation is a linear order if it is antisymmetric, transitive, and complete. Finally, we will say that it is a partial order if it is reflexive, antisymmetric, and transitive. For any $A \in \mathcal{D}, M(A, \succeq)$ denotes the set of maximal elements in $A$ with respect to $\succeq$, that is, $M(A, \succeq)=\{x \in A: y \succ x$ for no $y \in A\}$.

\footnotetext{
${ }^{2}$ To avoid tedious duplication of notation, we denote both the multi-valued and the singlevalued cases by $c$. In any case, the context will be specific enough to avoid confusion.
} 
Let $c(A) \succeq c(B), A, B \in \mathcal{D}$, denote the case when for every $x \in c(A)$ and $y \in c(B)$, we have $x \succeq y$.

The classic notion of rationalization of a choice correspondence deals with the issue of existence of a unique complete preorder relation $\succeq$ that explains choice behavior defined on $\mathcal{U}{ }^{3}$ The question is whether there is a $\succeq$ such that $c(A)=M(A, \succeq)$ for every $A \in \mathcal{U}$. A well-known result establishes that a choice correspondence $c$ satisfies WARP if and only if there is a complete preorder $\succeq$ such that $c(A)=M(A, \succeq)$ for every $A \in \mathcal{U}$. This result makes it clear that if $c$ does not satisfy WARP then a broader definition of rationalization is needed.

In the context of single-valued choice functions and universal choice domains $\mathcal{U}$, KRS propose the rationalization of any possible choice function through collections of linear orders. The interest is naturally directed to the minimal number of linear orders that rationalizes choice behavior. Here we use this notion of rationalization. To this end we extend the original definition to include choice correspondences and arbitrary choice domains $\mathcal{D}$. Accordingly, we also substitute linear orders by complete preorders.

Minimal Rationalization by Multiple Rationales (RMR): A $K$-tuple of complete preorders $\left(\succeq_{k}\right)_{k=1, \ldots, K}$ on $X$ is a rationalization by multiple rationales (RMR) of $c$ if for every $A \in \mathcal{D}$, there is a $k \in\{1, \ldots, K\}$, such that $c(A)=M\left(A, \succeq_{k}\right)$. It is said to be minimal if any other RMR of $c$ has at least $K$ preference relations.

Note that a minimal RMR is an extension of the classic idea of rationalization by one rationale. In fact, if $c$ satisfies WARP, then the minimal RMR is composed of one complete preorder. Further, note also that the case of choice correspondences generalizes the case of single-valued choice functions. The classic result in the latter context is that a single-valued choice function $c$ is rationalized by a linear order if and only if $c$ satisfies the property of independence of irrelevant alternatives (IIA):

Independence of Irrelevant Alternatives (IIA): For any $A, B \in \mathcal{D}$, if $y \in A \subseteq B$ and $y \in c(B)$, then $y \in c(A)$.

We highlight an especially important class of choice sets; the collection of $c$-maximal sets. These are the sets that must be explained in order to rationalize behavior. A set $S$ is $c$-maximal if any addition to $S$ of elements (consistent

\footnotetext{
${ }^{3}$ Uzawa (1957), Arrow (1959), Richter (1966) and Sen (1970) were among the first to study aspects of this problem.
} 
with the domain of choice problems $\mathcal{D}$ ) leads to a change in choice behavior with respect to the original elements of $S$. Formally,

$c$-Maximal Sets: A subset $S \in \mathcal{D}$ is said to be $c$-maximal if for all $T \in \mathcal{D}$, with $S \subset T$, it is the case that $c(S) \neq c(T) \cap S$. Denote the family of $c$ maximal sets under the choice domain $\mathcal{D}$ by $M_{c}^{\mathcal{D}}$.

For any $c$-maximal set there is no possibility of obtaining its associated elements from any other superset of it included in the domain $\mathcal{D}$, and thus, the study of choice behavior needs to incorporate it. ${ }^{4}$ Clearly, all other choice sets in $\mathcal{D}$ can be trivially associated with at least one $c$-maximal set.

2.2. NP-completeness. We now present an informal introduction to the notion of complexity we use in this paper. For an excellent, detailed and formal account see Garey and Johnson (1979). ${ }^{5}$

In theoretical computer science the computational complexity of a problem is measured by the relation between the size of the problem and the time required to solve it. There are different classes of problems. The P-class encompasses problems that can be solved in polynomial time. That is, a problem belongs to the $\mathrm{P}$-class if there is an algorithm that solves the problem, and the time required is upper bounded by a polynomial function of the size of the problem. More precisely, a time algorithm $f(t)$ is polynomial if there is a polynomial function $p$ such that $|f(t)| \leq|p(t)|$ for every $t \geq 0$, with $t$ denoting the size (or the input length) of the problem. In this sense we write that the complexity function of $f(t)$ is $O(p(t))$.

The NP-class is the family of problems that may be difficult to solve (it may take exponential time, or an even greater amount of time), although it is relatively easy (it takes polynomial time) to verify a particular instance of the problem (that is a concrete case where the parameters have been realized).

Within the NP-class there is one class of problems of particular interest. This is the class of NP-complete problems. The NP-complete problems are regarded as the most difficult problems in NP. This is because no polynomial time algorithm is known to solve any of them, but if a polynomial algorithm were found for one problem, then such an algorithm could be translated into polynomial time algorithms for all other problems in the NP-complete class. If an algorithm cannot be polynomially bounded, then it is typically called

\footnotetext{
${ }^{4}$ In the simpler case of single-valued choice functions, the condition to identify the class of $c$-maximal sets is simply $c(S) \neq c(T)$.

${ }^{5}$ See also Cormen, Leiserson, Rivest, and Stein (2001). Ballester (2004) and Aragones, Gilboa, Postlewaite and Schmeidler (2005) provide introductions in the context of economics.
} 
an exponential time algorithm. ${ }^{6}$ In this sense we say that only exponential time algorithms are known for NP-complete problems, and these problems are regarded as intractable. It is interesting to note that, although there is no known polynomial time algorithm solving an NP-complete problem, a proof showing that no such algorithm exists is still awaited. In fact, this is regarded as one of the major unsolved problems in mathematics.

The theory of NP-completeness is conventionally centered around decision problems. These are problems formulated with a yes-or-no answer. Consequently, we define a decision problem that is the binary analog of the problem of finding a minimal RMR of a choice correspondence $c$ on $\mathcal{D}$ as follows.

Rationalization by Complete Preorders in $\mathcal{D}(\mathbf{R C P}-\mathcal{D})$ : Given a choice correspondence $c$ on $\mathcal{D}$, can we find $k \leq K$ complete preorders that constitute a rationalization by multiple rationales of $c$ ?

Setting $\mathcal{D}=\mathcal{U}$, respectively, substituting complete preorders by linear orders, we define the binary problems $\mathrm{RCP}-\mathcal{U}$, Rationalization by Linear Orders in $\mathcal{D}(\mathrm{RLO}-\mathcal{D})$ and RLO- $\mathcal{U}$.

In this paper we use the proof-by-restriction technique to prove that a particular (decision) problem is NP-complete. That is, to prove that a given problem $\Pi$ in NP is in fact NP-complete, we show that it contains a known NP-complete problem $\Pi^{\prime}$ as a special case.

\section{Complexity of Rationalization}

We start with the classic, structured case, where $\mathcal{D}=\mathcal{U}$ and WARP holds. Let us call this case the rational procedure. Then we will study the polar case of the rational procedure. This is the case where no restriction whatsoever is imposed either on choice behavior, or on the choice domain. At the same time, we will deal with a number of intermediate cases.

3.1. Structured choice behavior: The rational procedure. We start by considering the case where a choice correspondence defined over the universal choice domain $\mathcal{U}$ satisfies WARP. We have already mentioned that this represents a very structured case, as it is well-known that there exists a unique complete preorder relation $\succeq$ rationalizing $c$.

Now the question arises of how difficult it is to find the rationalizing binary relation $\succeq$. Intuitively, it seems that the high degree of structure of the rational procedure implies that finding the rationale is not a difficult task. This is in

\footnotetext{
${ }^{6}$ However, note that this leads to classify algorithms that are $O\left(n^{\log n}\right)$ or $O(n !)$ as exponential.
} 
fact the case. It is easy to show that the set $\left|M_{c}^{\mathcal{U}}\right|$ is small, as it contains at most $n$ elements. The simplicity of the family of maximal sets allows us to consider very simple algorithms to obtain the rationalization of choice behavior in polynomial time. Consider for instance the following trivial one.

Take $X_{0}=X$ and iteratively define $X_{k}=X_{k-1} \backslash c\left(X_{k-1}\right)$ if $X_{k-1} \backslash c\left(X_{k-1}\right) \neq$ $\emptyset$ holds. Then, the set of maximal elements is the collection of sets $\left\{X_{k}\right\}$, with cardinality equal to or less than $n$. Clearly, every element is chosen from exactly one set $X_{k}$. Then, the rationale can be defined by stating that $x \succeq y$ if and only if $x \in X_{k}, y \in X_{j}$, with $X_{k}, X_{j}$ in the family of $c$-maximal sets $\left\{X_{k}\right\}$ and $k \leq j$.

We summarize the above in the following observation.

Observation 3.1. Let the choice correspondence $c$ be a rational procedure and $\mathcal{D}=\mathcal{U}$, then $\left|M_{c}^{\mathcal{U}}\right| \leq n$ and the problem of finding the complete preorder $\succeq$ that rationalizes $c$ is polynomial.

There are two important remarks to Observation 3.1. First, as a corollary to the above, finding the linear order $\succ$ rationalizing a single-valued choice function defined on $\mathcal{U}$ that satisfies IIA is also polynomial. This follows from the fact that the single-valued case is but a special case of the multi-valued choice correspondence.

Second, the results also hold for arbitrary choice domains $\mathcal{D}$. To find the binary relation rationalizing $c$ when $\mathcal{D} \neq \mathcal{U}$, simply write $x \succeq y$ if and only if $x \in c(A)$ and $y \in A, A \in \mathcal{D}$. It is easy to see that WARP guarantees that such a binary relation is a complete preorder.

3.2. Unstructured behavior and unrestricted domain. We now turn to the polar case of the rational procedure where we neither impose structure on choice behavior, nor on the domain of choice sets. This means that, in general, there is not a single binary relation rationalizing choice behavior, but a set of them in the sense of KRS. It is clear that the problem of finding the rationales in this case is a much more demanding task than the one we faced for the rational procedure in the previous section. In fact we show below that the task is demanding to the point of being intractable. That is, we show that finding a minimal RMR in this setting belongs to the class of NP-complete problems, and hence unless $\mathrm{P}=\mathrm{NP}$, there is no hope of finding an efficient algorithm that for every choice correspondence $c$ gives a minimal RMR in a reasonable time frame.

We can now state our first NP-complete result.

Theorem 3.2. $R C P-\mathcal{D}$ is NP-complete. 
Proof of Theorem 3.2: It is easy to show that RCP-D is in NP. A candidate for a solution consists of $k \leq K$ complete preorders that can be easily checked to rationalize the choice corrrespondence $c$.

Now consider the following problem that is known to be NP-complete.

Partition into Cliques (PIC): Given a graph $G=(V, E)$, can the vertices of $G$ be partitioned into $k \leq K$ disjoint sets $V_{1}, V_{2}, \ldots, V_{k}$ such that for $1 \leq i \leq k$ the subgraph induced by $V_{i}$ is a complete graph?

We now find a restriction of $\mathrm{RCP}-\mathcal{D}$ that makes it identical to PIC. First, let $H=\left\{h_{1}, \ldots, h_{|V|}\right\}$. Now, let $X$ be the union of the set of vertices $V$ and the set $H$. For each vertex $i \in V$ define the set $S_{i}=\{i\} \cup\{j: i j \notin E\} \cup\left\{h_{i}\right\}$ and denote $\mathcal{S}=\left\{S_{i}: i \in V\right\}$. Choice behavior is defined as follows. For every $S_{i} \in \mathcal{S}$ let $c\left(S_{i}\right)=i$. Clearly, this defines a single-valued choice function in $\mathcal{D}$, that is but a special case of a choice correspondence in $\mathcal{D}$. Note that the construction of $X, \mathcal{D}$, and $c$ can be done in polynomial time in the size of $|V|$.

We now prove that $G$ can be partitioned into less than $K$ cliques if and only if $c$ can be rationalized by less than $K$ rationales.

First, consider a collection $\left(\succeq_{p}\right)_{p=1, \ldots, k}$ with $k \leq K$ complete preorders rationalizing $c$. Consider a single-valued mapping (there may be several) $f: \mathcal{S} \rightarrow\{1, \ldots, k\}$ such that $f\left(S_{i}\right)=p$ if and only if $\succeq_{p}$ rationalizes the choice in $S_{i}$. Define $V_{p}=\left\{i: f\left(S_{i}\right)=p\right\}$. Clearly, the union of all sets $V_{p}$ is a partition of the vertex set $V$. Now, we show that $V_{p}$ is a clique. Otherwise, let $i, j \in V_{p}$ such that $i, j \notin E$. Then, $i, j \in S_{i} \cap S_{j}$ and hence, given the definition of $c$ over the class $\mathcal{S}$, for the rationalization of $S_{i}$ and $S_{j}$ it must hold that $i \succ_{p} j$ and $j \succ_{p} i$. But this contradicts that $\succeq_{p}$ is a complete preorder. We conclude that $V_{p}$ is a clique, as desired. Therefore the partition of $G$ is composed of at most $k$ cliques, and hence there is obviously a PIC of $G$ with at most $K$ cliques.

In the other direction, let $\left\{V_{p}\right\}_{p=1, \ldots, k}$ be a partition of $G$ into $k \leq K$ cliques. Define for every $p=1, \ldots, k$, the partial order $\succeq_{p}^{\prime}$ by: $x \succeq_{p}^{\prime} y$ if and only if $\left[x \in V_{p}\right.$ or $\left.y \in X \backslash V_{p}\right]$. Take any linear extension of $\succeq_{p}^{\prime}$ and denote it by $\succeq_{p}$. We now have to show that $\left\{\succeq_{p}\right\}_{p=1, \ldots, k}$ rationalizes all the selections. We claim that the collection of sets $S_{i}, i \in V_{p}$ is rationalized by $\succeq_{p}$. Otherwise, there would be a set $S_{i}$ with $i \in V_{p}$, and an element $w \in S_{i}, w \neq i$ with $w \succ_{p} i$. This implies that given the definition of $\succeq_{p}$, since $i \in V_{p}$, it must be $w \in V_{p}$. Since $V_{p}$ is a clique it must be that $(i, w) \in E$, but this contradicts that $w \in S_{i}$. This shows that there is a collection of at most $K$ complete preorders that 
rationalizes $c . \square$

Note that the $c$ used in the proof is single-valued and the binary relations defined from the partition of the graph are linear orders. Hence, the following corollary to (the proof of) Theorem 3.2 is immediate.

Corollary 3.3. $R L O-\mathcal{D}$ is NP-complete.

Theorem 3.2 (and Corollary 3.3) show that the conjunction of (i) unstructured choice behavior and (ii) unrestricted choice domain lead to the NPcompleteness of the problem of rationalization. But are the two conditions required to get the intractability result? In principle, it could be that the real difficulty in finding a minimal RMR is completely triggered by choice behavior per se, or it could be that it is the interplay of behavior and domain that drives the result. Theorem 3.4 below suggests that in the case of single-valued choice functions it is the interplay of both, unstructured behavior and unrestricted domain, that triggers the intractability of finding the rationales.

Theorem 3.4. $R L O-\mathcal{U}$ is quasi-polynomially bounded.

Proof of Theorem 3.4: Let $c$ be a single-valued choice function defined on $\mathcal{U}$, and consider the following naive algorithm. The naive algorithm would examine all the $k$-tuples of order relations, with $1 \leq k \leq n-2$. That is, it will start by checking whether there is one rationale that rationalizes behavior. If yes, the algorithm stops. Otherwise, it checks whether there are two rationales rationalizing behavior, and so on. Proposition 1 in KRS shows that the minimal book is composed of at most $n-1$ rationales. We now proceed to compute the order of magnitude of the algorithm. The main components of the input size and the number of operations are the number of choice sets and the number of collections of rationales to check. That is, since the choice domain is the universal one, the input size $x$ of the algorithm is roughly $2^{n}$. The naive algorithm requires a number of operations $t$ which is roughly $n !^{n-1}$. These values take into account only the number of choice problems $2^{n}$ and the set of possible collections of $n-1$ linear orders $n !^{n-1}$. Note that a more precise formulation would include other considerations such as the number of elements in each choice set, the chosen element, a checking protocol for the rationalization of choice, or the length of the data expressed in bits. It is not difficult to see, however, that these considerations have no influence on the final conclusion. Hence, we avoid these details here.

Now, to check the order of magnitude of the operations we relate $t$ to $x^{\alpha}$. This gives an $\alpha$ that is $O(\log n !)$ or equivalently $O(\log x \log \log x)$. Hence, it 
corresponds to the case where the naive algorithm has a quasi-polynomial order of magnitude of $O\left(x^{\log x \log \log x}\right)$. Therefore, there is a quasi-polynomially bounded algorithm that computes a minimal book for RCP- $\mathcal{U}$, wich completes the proof. $\square$

Theorem 3.4 suggests that RLO- $\mathcal{U}$ is not NP-complete. ${ }^{7}$ Otherwise, Theorem 3.4 would imply that all NP-complete problems are quasi-polynomially bounded. However, in spite of continuous efforts such a bound has never been found for NP-complete problems. Indeed, there is the strong conviction this will never happen.

The question with regard to choice correspondences defined on $\mathcal{U}$, however, remains open. The naive algorithm used in Theorem 3.2 is not quasipolynomially bounded in this case. It is not difficult to see that the number of possible complete preorders is upper bounded by $n !^{2}$. The problem arises because, in the case of complete preorders, the $n-1$ bound on the maximum number of preference relations to check for rationalization does not hold. In fact, it is straightforward to see that the bound turns out to be $2^{n}$. This implies that the naive algorithm for choice correspondences cannot be quasipolynomially bounded, having an exponential order of magnitude. Therefore, it may well be the case that with choice correspondences, the practical difficulty in finding a minimal RMR is triggered by choice behavior per se.

The challenge for future research is then to understand the driving force of the complexity of rationalization, and use this understanding to find specific algorithms that behave well under certain circumstances. In the next section we start this task by drawing a connection with graph theory. This is especially useful since there is a wealth of algorithms for graph problems that may solve the problem of rationalization of certain choice structures.

\section{ON THE STRUCTURE OF THE COMPLEXITY OF RATIONALIZATION}

4.1. Rationalization and graph theory. The following binary relations capture two fundamental properties on the structural relation of maximal sets.

Definition 4.1. Let $A, B \in M_{c}^{\mathcal{D}}, A \rightarrow B$ if and only if $c(A) \cap B \notin\{\emptyset, c(B) \cap A\}$.

This first binary relation $\rightarrow$ states the conditions under which a set $A$ blocks another set $B$, in the sense that $A$ and $B$ cannot be rationalized by a binary

\footnotetext{
${ }^{7}$ The naive algorithm of Theorem 3.4 does not necessarily behave well in unrestricted choice domains $\mathcal{D}$. The reason being that in this case the input size need not be as high as $2^{n}$, but for example, it could be $n$. This gives an exponential order of magnitude for the naive algorithm.
} 
relation $\succeq$ writing $c(A) \succeq c(B)$. Define $H_{A}=A \backslash c(A), A \in \mathcal{D}$. Then, when $c$ is single-valued, $\rightarrow$ reduces to: $A \rightarrow B$ if and only if $c(A) \in H_{B}$.

Definition 4.2. Let $A, B \in M_{c}^{\mathcal{D}}, A-B$ if and only if $c(A) \cap c(B) \neq \emptyset$.

$A-B$ means that sets $A$ and $B$ are linked in the rationalization problem. That is, if $A$ and $B$ are to be rationalized by the same binary relation $\succeq$ when $A-B$, then it must be that $c(A) \sim c(B)$.

Finally, we use the above two binary relations to define an oriented cycle. if

Oriented Cycle: The collection $\left\{A_{t}\right\}_{t=1}^{n} \in M_{c}^{\mathcal{D}}, n \geq 2$, is an oriented cycle

(1) $A_{1}=A_{n}$,

(2) for every $i \in\{1, \ldots, n-1\}$, either $A_{i} \rightarrow A_{i+1}$, or $A_{i}-A_{i+1}$, and

(3) there is $j \in\{1, \ldots, n-1\}$ such that $A_{j} \rightarrow A_{j+1}$.

We are now in a position to introduce a graph theory problem over the space of $c$-maximal sets.

Minimal Non-Oriented Partition (NOP): A partition of $M_{c}^{\mathcal{D}}\left\{V_{p}\right\}_{p=1, \ldots, P}$ is said to be a non-oriented partition if for every class $V_{p}$ there is no oriented cycle. It is said to be minimal if any other NOP has at least $P$ classes.

A (minimal) NOP is constructed over the set $M_{c}^{\mathcal{D}}$ according to the binary relations $\rightarrow$ and - . Hence, a class $V_{p}$ in the partition has a clear interpretation: all the sets in the class $V_{p}$ can be rationalized through a complete preorder $\succeq_{p}$. Then, an NOP gives information on which choice problems can be rationalized together.

The following theorem establishes finding a minimal RMR is equivalent to finding a minimal NOP. This opens the possibility of drawing upon the established algorithm knowledge on graph theory problems.

Theorem 4.3. Let c be a choice correspondence:

-If $\left\{\succeq_{p}\right\}_{p=1, \ldots, P}$ is a minimal $R M R$, then there is a minimal NOP $\left\{V_{p}\right\}_{p=1, \ldots, P}$.

-If $\left\{V_{p}\right\}_{p=1, \ldots, P}$ is a minimal NOP, then there is a minimal $R M R\left\{\succeq_{p}\right\}_{p=1, \ldots, P}$.

Proof of Theorem 4.3: Let $\left\{\succeq_{p}\right\}_{p=1, \ldots, P}$ be an RMR of $c$. Consider a singlevalued mapping (there may be several) $f: M_{c}^{\mathcal{D}} \rightarrow\{1, \ldots, P\}$ such that $f(A)=$ $j$ if and only if $\succeq_{j}$ rationalizes the choice in $A$. The mapping $f$ naturally induces a partition of $M_{c}^{\mathcal{D}}$ containing at most $P$ classes. Let $V_{p}=\left\{A_{p}^{s}\right\}_{s=1,2, \ldots, S_{p}}$ be the $c$-maximal sets associated with class $p, 1 \leq p \leq P$ (some of them may be empty). We now show that there is no oriented cycle in $V_{p}$. Assume the 
contrary is the case. That is let $\left\{A_{t}\right\}_{t=1}^{n} \in M_{c}^{\mathcal{D}}, n \geq 2$ be an oriented cycle in $V_{p}$. Then, by definition, there is $j \in\{1, \ldots, n-1\}$ such that $A_{j} \rightarrow A_{j+1}$, and hence $c\left(A_{j}\right) \cap A_{j+1} \notin\left\{\emptyset, c\left(A_{j+1}\right) \cap A_{j}\right\}$.

Assume that $c\left(A_{j+1}\right) \cap A_{j} \neq \emptyset$. Then $A_{j+1} \rightarrow A_{j}$ and there must be an element $x \in X$ such that either $x \in c\left(A_{j}\right) \cap H_{A_{j+1}}$ or $x \in c\left(A_{j+1}\right) \cap H_{A_{j}}$. Without loss of generality, assume the first case. Then for $\succeq_{p}$ to rationalize $A_{j+1}$ it must be $c\left(A_{j+1}\right) \succ_{p} x$. Since $c\left(A_{j+1}\right) \cap A_{j} \neq \emptyset$, there is $y \in A_{j}$ such that $y \succ_{p} x$. But this contradicts the rationalization of $A_{j}$ since $x \in c\left(A_{j}\right)$. Hence, it must hold that $c\left(A_{j+1}\right) \cap A_{j}=\emptyset$, and therefore, for $\succeq_{p}$ to rationalize $A_{j}$ and $A_{j+1}$ it must hold $c\left(A_{j+1}\right) \succ_{p} c\left(A_{j}\right)$.

Now, consider $A_{j+2}\left(A_{2}\right.$ if $\left.A_{j+1}=A_{n}=A_{1}\right)$. Since, by assumption, it is an oriented cycle, it must be either that $A_{j+1} \rightarrow A_{j+2}$ or $A_{j+1}-A_{j+2}$. In the first case, the argument in the previous paragraph shows that $c\left(A_{j+2}\right) \succ_{p} c\left(A_{j+1}\right)$. In the second case, it must obviously hold that $c\left(A_{j+2}\right) \sim_{p} c\left(A_{j+1}\right)$. In both cases, by the transitivity of $\succeq_{p}$ it must be that $c\left(A_{j+2}\right) \succ_{p} c\left(A_{j}\right)$. Applying this reasoning iteratively we conclude that $c\left(A_{j}\right) \succ_{p} c\left(A_{j}\right)$, which is of course absurd. This shows that there is no oriented cycle.

Now take an NOP $\left\{V_{p}\right\}_{p=1, \ldots, P}$. For every class $V_{p}$ we construct a rationale $\succeq_{p}$ that rationalizes all the elements in the class $V_{p}$. We start by defining an equivalence relation $E$ between the elements of $V_{p}$ : $A E B$ if and only if there is a chain of elements in $V_{p}$ such that $A=A_{1}-A_{2}-\cdots-A_{q}=B$. The equivalence relation $E$ partitions $V_{p}$ into disjoint classes $\left\{L_{p}^{i}\right\}_{i=1}^{I_{p}}$. We write $L_{p}^{i} \rightarrow L_{p}^{j}$ when there are $A \in L_{p}^{i}$ and $B \in L_{p}^{j}$ with $A \rightarrow B$.

We start by noting that there must be at least one class, denoted by $L_{p}^{1}$, such that $L_{p}^{1} \rightarrow L_{p}^{i}$ holds for no $L_{p}^{i}, i>1$. Otherwise, due to the finiteness of $X$ and hence to the number of classes $L_{p}^{i}$ in $V_{p}$, there would be a cycle. Using the same argument there must be another class $L_{p}^{2}$ such that $L_{p}^{2} \rightarrow L_{p}^{i}$ holds for no $L_{p}^{i}, i>2$. Clearly, this argument extends to all classes $\left\{L_{p}^{i}\right\}_{i=1}^{I_{p}}$. We write $c\left(L_{p}^{i}\right)=\cup_{A \in L_{p}^{i}} c(A)$ and $c\left(V_{p}\right)=\cup_{A \in V_{p}} c(A)$. Now, we show that the order

$$
c\left(L_{p}^{1}\right) \succ_{p} c\left(L_{p}^{2}\right) \succ_{p} \cdots \succ_{p} c\left(L_{p}^{I_{p}}\right) \succ_{p} X \backslash c\left(V_{p}\right)
$$

rationalizes $V_{p}$. Assume the contrary is the case. Let $j \in\left\{1, \ldots, I_{p}\right\}$ and $A \in L_{p}^{j}$ with $y \in H_{A}$ but $y \succeq_{p} c(A)$. By construction of $\succeq_{p}$ this means that $y \in c(B)$ for some $B \in L_{p}^{k}, k \leq j$. From $y \in H_{A} \cap c(B)$, obviously $c(B) \cap A \notin\{\emptyset, c(A) \cap B\}$ and, hence, $B \rightarrow A$. By construction of the equivalence classes, it can only be $k=j$. But, since $A$ and $B$ belong to the same equivalence class there is a chain of elements $A=A_{1}-A_{2}-\ldots-A_{q}=B$ and, therefore, 
together with $B \rightarrow A$, it implies that there is an oriented cycle. This is absurd and therefore $\succeq_{p}$ rationalizes $V_{p}$.

Finally, to end the claims of the theorem we have to show the conditions on minimality. Then, for the first claim suppose that $\left\{\succeq_{p}\right\}_{p=1, \ldots, P}$ is minimal while $\left\{V_{p}\right\}_{p=1, \ldots, P}$ is not. Then there is an NOP with $T$ number of classes, $T<P$. But then, we have shown that there is an RMR with $T$ classes, contradicting the minimality of $\left\{\succeq_{p}\right\}_{p=1, \ldots, P}$. The reverse direction of the proof is analogous. This concludes the proof. $\square$

The significance of Theorem 4.3 is best appreciated in the case of singlevalued choice functions. Recall that when $c$ is single-valued, $A \rightarrow B$ if and only if $c(A) \in H_{B}, A, B \in M_{c}^{\mathcal{D}}$. That is, $A$ blocks $B$ if and only if the chosen element in $A$ belongs to $B$ and, at the same, time this element is not chosen in $B$. It is clear that such a case is inconsistent with a linear order rationalizing $B$ and writing $c(A) \succ c(B)$. Also, note that $A-B$ if and only if $c(A)=c(B)$. Then the relation between minimal RMRs and minimal NOPs simplifies considerably. This is because for any three sets $A_{1}, A_{2}, B \in M_{c}^{\mathcal{D}}$, whenever $c\left(A_{1}\right)=c\left(A_{2}\right)$, then $A_{1} \rightarrow B$ if and only if $A_{2} \rightarrow B$. This implies that, whenever there is an oriented cycle, there is a cycle composed of elements related only through $\rightarrow$. Hence the analysis can obviate the equivalence relation - , and focus on $\left(M_{c}^{\mathcal{D}}, \rightarrow\right)$. The latter is simply an standard directed graph, and the structure defined as an oriented cycle reduces to the standard notion of a directed cycle. There is an immense literature on graphs without directed cycles, typically known as directed acyclic graphs (DAGs). ${ }^{8}$ This is especially important since Theorem 4.3 guarantees that there is much to gain from the results in this literature. Hence, our problem reduces to find a minimal partition into DAGs.

4.2. An example. The study of concrete choice procedures appears particularly appealing. It is likely that the structure inherent to specific choice procedures allows for the tractability of rationalization. Here we provide an example that draws on the previous subsection.

Manzini and Mariotti (2007) study the nature of choice functions defined on $\mathcal{U}$ that can be rationalized by sequentially applying a fixed set of asymmetric binary relations in a universal domain. Among other results, they provide a full characterization of the case when a choice function is sequentially rationalizable by two rationales. Such a choice function is called a Rational Shortlist Method (RSM). Their characterization makes use of the classical property of

\footnotetext{
${ }^{8}$ See, e.g., Cormen, Leiserson, Rivest, and Stein (2001).
} 
expansion.

Expansion: If $x \in c(A)$ and $x \in c(B), A, B \in \mathcal{U}$, then $x \in c(A \cup B)$.

It is not difficult to observe that the set $M_{c}^{\mathcal{U}}$ shrinks considerably whenever this property holds. Clearly, for each element $x$ in $X$ there is at most one element in $M_{c}^{\mathcal{U}}$ for which $x$ is the chosen element, namely, the union of all subsets $A$ for which $x$ is chosen. Denote the latter by $M(x)$. Then the problem of finding a minimal RMR here reduces to finding a minimal partition into DAGs over the universal set of alternatives $X$ according to $\rightsquigarrow$, where for every $x \neq y$,

$$
x \rightsquigarrow y \text { if and only if } M(x) \rightarrow M(y) \text { if and only if } x \in M(y)
$$

Hence the rationalization of RSMs through multiple rationales turns out to be a computationally tractable problem.

\section{FINAL REMARKS}

We have used the tools of theoretical computer science to study the complexity of finding the preference relations that rationalize choice behavior. The question of rationalizability of choice behavior has played a central role in economics. However, surprisingly enough, virtually no attention has been given to the practical problem of computing the rationales.

We have shown that, in the classical case, when the weak axiom of revealed preference holds, finding the preference relation rationalizing choice behavior is easy. There are polynomial time algorithms that compute the rationale quickly. On the other hand, when we neither impose any restriction on choice behavior nor on the domain, we have shown that the problem of rationalization is NP-complete. Therefore, there is little hope of finding an efficient algorithm bounded above by a a polynomial function. Furthermore, we have shown that in the case of single-valued choice functions, it is the conjunction of unstructured choice and unrestricted domain that drives the intractability result. Under the universal domain, the problem of finding a minimal book is quasi-polynomially bounded. On the other hand, we argue that in the choice correspondences case, it may well be the case that the difficulty in finding a minimal book is triggered by choice behavior per se.

We then turned to trying to better understand the complexity of rationalization. To this end we identified two binary relations over choice sets that capture part of the essence of rationalization. Furthermore, these binary relations define a problem in graph theory that is equivalent to the problem of rationalization. This is particularly interesting since the complexity issues 
have attracted a great deal of attention in graph theory. The equivalence result provided allows for the searching of existing algorithms in graph theory that can be used for the problem of rationalization.

Apart from the literature on rationalization, our results relate to two other strands in the literature. First, the problem of rationalization can be read as the problem of transmission of information (choice behavior in different situations), given a specific grammar (complete preorders a la KRS). Under this interpretation, our paper is related to the economics literature on language (see Rubinstein, 2000). Rubinstein stresses the importance of binary relations to natural language. In this sense, our results establish that there are practical limitations to the design of a grammar with the ability to transmit any kind of information. Several questions arise. Does the structure of natural speech imply the existence of a collection of complete preorders computable in polynomial time? What, if anything, is lost in the transmission of information, if the problem of constructing a grammar is upper bounded by a polynomial time algorithm?

Second, an immediate conclusion from our results is that the more structured behavior is, the easier it is to rationalize it in practice. That is, rationality makes things easier. This type of observation has been recognized in the bounded rationality literature from different perspectives. Tversky and Simonson (1993) note that the standard maximization problem is hard to beat in terms of its simplicity of formulation. It is most likely that any descriptive bounded rationality model is condemned to involve a more cumbersome formulation. Also, Salant (2003) shows that the rational procedure requires the least memory possible and that the automatation required to compute the rational procedure is the smallest possible.

\section{REFERENCES}

[1] Aragones, E., I. Gilboa, A. Postlewaite, and D. Schmeidler (2005), "Fact-Free Learning," American Economic Review, 95:1355-1368

[2] Arrow, K.J. (1959), "Rational Choice Functions and Orderings," Economica, 26:121127.

[3] Ballester, C. (2004), "NP-completeness in Hedonic Games," Games and Economic Behavior, 49:1-30.

[4] Cormen, T.H., C.E. Leiserson, R.L. Rivest, and C. Stein (2001), Introduction to Algorithms. MIT Press.

[5] Garey, M.R. and D.S. Johnson (1979), Computers and Intractability: A Guide to the Theory of NP-Completeness. Freeman.

[6] Kalai, G., A. Rubinstein, and R. Spiegler (2002), "Rationalizing Choice Functions by Multiple Rationales," Econometrica, 70:2481-88. 
[7] Manzini, P. and M. Mariotti (2007), "Sequentially Rationalizable Choice," American Economic Review, forthcoming.

[8] Ok, E.A. (2005), "Independence of Irrelevant Alternatives and Individual Choice," mimeo, New York University.

[9] Richter, M.K. (1966), "Revealed Preference Theory," Econometrica, 34:635-645.

[10] Rubinstein, A. (2000), Economics and Language, Cambridge University Press.

[11] Salant, Y. (2003), "Limited Computational Resources Favor Rationality," mimeo, Hebrew University.

[12] Sen, A.K. (1971), "Choice Functions and Revealed Preferences," Review of Economic Studies, 38:307-317.

[13] Tversky, A. and I. Simonson (1993), "Context-dependent Preferences," Management Science, 39:1179-1189.

[14] Uzawa, H. (1957), "Notes on Preference and the Axiom of Choice," Annals of the Institute of Statistical Mathematics, 8:35-40.

[15] Xu, Y. and L. Zhou (2007) "Rationalizability of Choice Functions by Game Trees," Journal of Economic Theory, 134:548-556. 